\title{
A dynamo mechanism as the potential origin of the long cycle in double periodic variables
}

\author{
Dominik R. G. Schleicher and Ronald E. Mennickent
}

\begin{abstract}
Departamento de Astronomía, Facultad Ciencias Físicas y Matemáticas, Universidad de Concepción, Av. Esteban Iturra s/n Barrio Universitario, Casilla 160-C, Concepción, Chile e-mail: dschleicher@astro-udec.cl
\end{abstract}

Received 11 May 2016 / Accepted 9 March 2017

\begin{abstract}
The class of double period variables (DPVs) consists of close interacting binaries, with a characteristic long period that is an order of magnitude longer than the corresponding orbital period, many of them with a characteristic ratio of approximately 35 . We consider here the possibility that the accretion flow is modulated as a result of a magnetic dynamo cycle. Due to the short binary separations, we expect the rotation of the donor star to be synchronized with the rotation of the binary due to tidal locking. We here present a model to estimate the dynamo number and the resulting relation between the activity cycle length and the orbital period, as well as an estimate for the modulation of the mass transfer rate. The latter is based on Applegate's scenario, implying cyclic changes in the radius of the donor star and thus in the mass transfer rate as a result of magnetic activity. Our model is applied to a sample of 17 systems with known physical parameters, 11 of which also have known modulation periods. In spite of the uncertainties of our simplified framework, the results show a reasonable agreement, indicating that a dynamo interpretation is potentially feasible. At the same time, we note that the orbital period variations resulting from Applegate's model are sufficiently small to be consistent with the data. We conclude that both larger samples with known physical parameters as well as potential direct probes of the magnetism of the donor star, including cold spots as well as polarization, will be valuable to further constrain the nature of these systems.
\end{abstract}

Key words. binaries close - stars: variables: general - stars: activity - dynamo

\section{Introduction}

The study of interacting binary systems is particularly important as a probe of stellar physics and to understand the evolution of close binaries. An important class of these systems includes the Algol-type variables, which are semi-detached binary systems of intermediate stellar masses. As originally explained by Crawford (1955) and later confirmed via evolutionary calculations by Kippenhahn \& Weigert (1967), the mass ratio distribution between the evolved star and the main-sequence star indicates that severe interactions must have taken place in the binary system to account for the higher mass of the main-sequence star, including significant amounts of mass transfer (see also Sarna 1993; van Rensbergen et al. 2011; de Mink et al. 2014). After fast mass exchange as a result of Roche lobe overflow, the lobefilling donor star is thus significantly less massive than the primary (Eggleton \& Kisseleva-Eggleton 2006).

A particularly interesting feature in the interacting semidetached binaries is the observation of long cycles. The presence of such cycles is known since a long time, as Gaposchkin (1944) inferred the presence of a long cycle in RX Cas, Lorenzi (1980a,c) demonstrated the presence of a long cycle in AU Mon, and Guinan (1989) inferred it for $\beta$ Lyr. Nowadays, much larger samples of binaries with long cycles are known, and the above-mentioned systems as well as other binaries with wellknown physical parameters were for instance recently revisited by Harmanec et al. (2015).

The interpretation of these long cycles however remains enigmatic until this point. For instance, Peters (1994), associated the long-period variation of AU Mon to changes in the mass transfer rate due to cyclic pulsations of the donor star, but no compeling reason for this possible oscillation is provided. In this paper, our main concern are the double periodic variables (DPVs), a sub-class of the Algols consisting of particularly massive stars, where the primary has a typical mass of at least $\sim 7 M_{\odot}$, and the secondary is a star filling its Roche lobe. This class of binary stars was initially discovered based on the inspection of large scale photometric surveys of emission-line objects in the Magellanic Clouds. Mennickent et al. (2003) published a first list with initially 30 objects exhibiting roughly sinusoidal periodic light variations with periods from 140 to 960 days. They further showed periodic changes with periods from 2.4 to 15.9 days, including sinusoidal, ellipsoidal and eclipsing light curves. They found a characteristic relation between the long and short period of about $P_{\text {long }}=35.17 \times P_{\text {short }}$. This relation and the existince of the two periods has given rise to the name of double periodic variables.

The first spectroscopic data for a sample of DPVs in the Magellanic Clouds was obtained by Mennickent et al. (2005), who found that the optical spectrum was dominated by Balmer and helium absorption lines and a continuum with a blue or sometimes flat slope. One object in the sample showed a characteristic shortening of the long cycle by about $20 \%$ in some of the cycles, suggesting that the periodicity is not strict. Similarly, Mennickent et al. (2006) examined OGLE and MACHO light curves of DPVs spanning $11 \mathrm{yr}$, finding excursions from strictly periodic variability and a number of cases with either increasing or decreasing cycle lengths. Since then, additional DPV systems have been found both in the Galaxy (Mennickent et al. 2012a), in the Magellanic Clouds (Poleski et al. 2010; Pawlak et al. 2013) 
as well as toward the Galactic bulge (Soszyński et al. 2016). Interestingly, deeper primary eclipses are observed during the long cycle and in one system the secondary eclipse disappears at some epochs (Poleski et al. 2010).

The DPV OGLE05155332-6925581 is a semi-detached system with $\mathrm{H} \alpha$ emission and a luminous accretion disk similar to $\beta$ Lyr; a preliminary interpretation was given for their long cycle in terms of cycles of mass loss in the system, probably feeding a circumbinary disk (Mennickent et al. 2008), a kind of decretion disk as described by Tutukov \& Pavlyuchenkov (2004). A similar explanation was put forward by Desmet et al. (2010) in the context of AU Mon, challenging the Peters (1994) interpretation; CoRoT space photometry shows that the orbital light curve remains practically constant through the long cycle. Other explanations that have previously been considered include the potential influence of disk winds, as detected for instance in V 393 Sco (Mennickent et al. 2012b) and HD 170582 (Mennickent et al. 2015). Similarly, the precession of the circumprimary disk could, in principle, lead to such effects via a 3:1 resonance (Mennickent et al. 2003), but the observed double emission lines show no such indications. However, the relation to the cool donor star became more obvious from the four-colour light curves for several DPVs, published by Michalska et al. (2010) showing that in all cases the amplitudes of the long-term cyclic changes were growing toward longer wavelengths.

In a recent study, Mennickent et al. (2016a) attempt to further clarify the physical origin of the DPV phenomenon as well as the nature of their disks, which were frequently inferred around the more massive stars. As it is well known from the studies by Kříž $(1970,1971,1972)$, there is an important distinction between direct impact in shorter period systems, and the formation of an accretion disk if the impact parameter is initially larger than the radius of the primary. Lubow \& Shu (1975) quantified this criterion in terms of the Lubow-Shu critical radius. If the radius of the primary is smaller than this critical radius, it means that an accretion disk will form around the star, while the absence of a disk is expected if the stellar radius is larger than the critical radius, and the accretion flow will then directly hit the stellar surface. Mennickent et al. (2016a) found that the critical radius appears to be very similar to the radii of the primaries for the known DPV systems, suggesting that the accretion flow hits the surface of the primary in an almost tangential manner.

This latter allows us, in particular, to distinguish the DPVs from the class of W Serpentis stars which include similar stellar masses and orbital periods, but where the radius of the primary is typically much smaller than the critical radius. This class of stars consists of a hot primary surrounded by an optically thick accretion disk (Plavec 1980; Young \& Snyder 1982), and is characterized by strong ultraviolet emission lines of highly excited species, including He II, C II, Al III, Fe III, C IV, Si IV and N V, potentially formed in a super corona produced via mass transfer and accretion (Plavec et al. 1982; Plavec 1989). They show significant variations of the orbital period and appear to be strongly interacting.

In the following, we aim to clarify the overall picture of the DPVs that has emerged over time. They consist of a B-type star plus a less massive $\left(1-3 M_{\odot}\right)$ companion filling the Roche lobe, leading to mass overflow to the more massive star. The more massive star is surrounded by a massive and optically thick disk, where the disk radius is comparable to the radius of the primary star. Based on calculations following the evolutionary tracks of these systems (Mennickent et al. 2016a), it appears very likely that the primary has accreted a significant mass from the donor, thereby gaining a large amount of angular momentum. Even if not expected within the Lubov-Shu picture, the formation of an accretion disk is thus plausible, as likely not all of the angular momentum can be accreted onto the primary star, and a mechanism like a disk wind may thus be beneficial to loose some of the angular momentum. The eccentricity in DPVs is generally compatible with zero; in very few cases it is very small and consistent with the effects of the mass streams (Lucy 2005). The latter is expected as a result of rapid circularization via dynamical tides (Tassoul 1987; Zahn 1989; Zahn \& Bouchet 1989).

In this paper, we consider magnetic activity cycles as a potential origin of the long period in DPVs. These long cycles have been inferred via changes in the light curves, rather than orbital period variations and we will, in fact, show that the expected orbital period variations are negligible for the DPVs. In the following, we provide a brief overview on what is known regarding magnetic activity in different types of binary systems. In fact, already the Algol system itself shows a characteristic $32 \mathrm{yr}$ cycle originally reported by Soderhjelm (1980), and its connection to magnetic activity has been confirmed via radio observations by Peterson et al. (2010). The possibility of such magnetic activity cycles has been put forward by Sarna et al. (1997) for the more general class of Algol-type systems, and was more recently revisisted by Soker (2002).

In the sub-class of RS CVn stars, which are detached binaries typically composed of a chromospherically active $G$ or $\mathrm{K}$ star, the orbital period variations were explored by Lanza et al. (1998) and Lanza \& Rodonò (1999) and related to the presence of a magnetic activity cycle. The latter can be explained with Applegate's mechanism, explaining the orbital period variations as a result of quasi-periodic changes of the quadrupole moment due to the internal redistribution of angular momentum via magnetic fields (Applegate \& Patterson 1987; see Völschow et al. 2016; for an updated version of the formalism). As further described by Bolton (1989) and Meintjes (2004), the presence of a dynamo may modulate the mass transfer rate in Algol systems, leading to a characteristic impact of the dynamo cycle on the luminosity of the resulting hot spot (even if there is not a hot spot, but rather a hot belt, as proposed by Bisikalo \& Kononov 2010, the resulting behavior will be similar).

Orbital period modulations have also been observed for a significant number of post-common-envelope binaries (PCEBs), including V471 Tau, DP Leo and QS Vir (Zorotovic \& Schreiber 2013). While there is still an ongoing debate over whether planets might also be present in these systems, at least for some of them the presence of magnetic activity is clearly confirmed. For V471 Tau, magnetic activity was shown via photometric variability, flaring events and $\mathrm{H} \alpha$ emission along with a strong X-ray signal (Kamiński et al. 2007; Pandey \& Singh 2008). For DP Leo, the presence of magnetic activity is indicated via X-ray observations (Schwope et al. 2002), and in case of QS Vir, it has been inferred via detections of Ca II emission and Doppler Imaging (Ribeiro et al. 2010) as well as observed coronal emission (Matranga et al. 2012). A theoretical model for dynamos in these type of systems was put forward by Rüdiger et al. (2002).

Even for single main-sequence stars, characteristic relations between the stellar rotation period and the dynamo cycle are known, and have been reported by Saar \& Brandenburg (e.g. 1999), Böhm-Vitense (e.g. 2007). These results are frequently interpreted in terms of dynamo models, as already Soon et al. (1993) and Baliunas et al. (1996) suggested a correlation between rotation velocity, activity period and dynamo number $D=\alpha \Delta \Omega d^{3} / \eta^{2}$. Here $\alpha$ is a measure of helicity, $\Delta \Omega$ the large-scale differential rotation, $d$ the characteristic length scale of convection and $\eta$ the turbulent magnetic diffusivity in the 
star. Beyond the main sequence, successful dynamo simulations have been pursued for AGB stars by Blackman et al. (2001) and Dorch (2004), while polarized water maser observations have confirmed the presence of $\sim 10-100 \mathrm{G}$ magnetic fields around evolved isolated stars (Vlemmings et al. 2002, 2005; Leal-Ferreira et al. 2013). In case of the asymptotic giant branch star W43A, Vlemmings et al. (2006) further detected the presence of a collimated jet as a result of magnetic activity.

Our paper is structured as follows: In sect. 2, we present a dynamo model for DPVs including the formalism to estimate the dynamo number. Section 3 presents a mechanism based on Applegate's scenario to explain a time-dependent mass transfer as a result of the dynamo cycle. In Sect. 4, we compare our models to a sample of currently discussed DPV candidates. A final discussion is given in Sect. 5.

\section{A dynamo model for DPVs}

In the context of dynamo models, the dynamo cycle $P_{\text {cycle }}$ is related to the rotation period $P_{\text {rot }}$ via a relation of the form (Soon et al. 1993; Baliunas et al. 1996)

$P_{\text {cycle }}=D^{\alpha} P_{\text {rot }}$,

with $D$ the dynamo number and $\alpha$ a power-law index, with typical values of $\alpha$ between $\sim \frac{1}{3}$ and $\sim \frac{5}{6}$. Observational studies of single main-sequence stars have pointed toward an index $\alpha \sim 0.25$ for two parallel branches (Saar \& Brandenburg 1999), i.e. implying two different normalizations, while recent simulations by Dubé \& Charbonneau (2013), along with data for BY Dra and W UMa, hint at the possible existence of a super-active branch with $\alpha \sim-0.2$. The precise value of the power-law index is thus still controversial, and may also depend on the astrophysical object under consideration. In the following, we will thus aim to explore the dependence of the dynamo number $D$ on the stellar parameters, while the power-law index $\alpha$ will be determined by comparison with a sample of available objects.

For this purpose, we express the dynamo number $D$ in terms of the Rossby number Ro as $D=\mathrm{Ro}^{-2}$. Followig Soker (2000), the latter is given as

Ro $=9\left(\frac{v_{\mathrm{c}}}{10 \mathrm{~km} \mathrm{~s}^{-1}}\right)\left(\frac{H_{\mathrm{p}}}{40 R_{\odot}}\right)^{-1}\left(\frac{\omega}{0.1 \omega_{\mathrm{Kep}}}\right)^{-1}\left(\frac{P_{\mathrm{Kep}}}{\mathrm{yr}}\right)$,

with $v_{\mathrm{c}}$ the convective velocity, $H_{\mathrm{p}}$ the pressure scale height, $\omega$ the angular velocity, $\omega_{\text {Kep }}$ the Keplerian angular velocity and $P_{\text {Kep }}$ the Keplerian orbital period of a test particle on the surface of the donor star. Assuming tidal locking (Tassoul 1987; Zahn 1989; Zahn \& Bouchet 1989), the angular velocity of the donor star is equal to the angular velocity of the binary. We thus have

$\omega=\sqrt{\frac{G\left(M_{1}+M_{2}\right)}{a^{3}}}$,

where $a$ denotes the separation of the binary, and we assume a circular orbit. As the radius of the donor stars fills the Roche lobe, it can be evaluated as (Paczyński 1971)

$R_{\text {Roche }}=0.46224 a\left(\frac{M_{2}}{M_{1}+M_{2}}\right)^{1 / 3} \propto a\left(\frac{1}{1+q^{-1}}\right)^{1 / 3}$

for $q=M_{2} / M_{1}<0.8$. We assume that the scale height $H_{\mathrm{p}}$ can be described as a fraction $\epsilon_{\mathrm{H}}$ of the Roche radius $R_{\text {Roche }}$. The
Keplerian angular velocity on the surface of the donor star then follows as

$\omega_{\mathrm{Kep}}=\sqrt{G M_{2} / R_{2}^{3}} \sim 3.2 \omega$,

implying $\omega \sim 0.31 \omega_{\text {Kep }}$. The Kepler period of a test particle at the surface of the donor star is

$P_{\mathrm{Kep}}=\frac{2 \pi}{\omega_{\mathrm{Kep}}}=0.31 \times \frac{2 \pi}{\omega}=0.31 \times 2 \pi \sqrt{\frac{a^{3}}{G\left(M_{1}+M_{2}\right)}} *$.

A central ingredient to evaluate the Rossby number is then the convective velocity $v_{\mathrm{c}}$. Under conditions of convective energy transport, it can be shown that (Kippenhahn et al. 2012)

$v_{\mathrm{c}}=v_{\mathrm{s}} \sqrt{\nabla-\nabla_{\mathrm{ad}}}$,

with $v_{\mathrm{S}}$ the speed of sound in the stellar interior, $\nabla=\frac{\mathrm{d} \ln T}{\mathrm{~d} \ln r}$ the physical temperature gradient and $\nabla_{\mathrm{ad}}=\left(\frac{\mathrm{d} \ln T}{\mathrm{~d} \ln r}\right)_{\mathrm{ad}}$ the temperature gradient under adiabatic conditions. From mixing length theory, we have

$F_{\text {conv }}=\rho c_{\mathrm{P}} T\left(\frac{l_{\mathrm{m}}}{H_{\mathrm{p}}}\right)^{2} \sqrt{\frac{1}{2} g H_{\mathrm{p}}}\left(\nabla-\nabla_{\mathrm{ad}}\right)^{3 / 2}$,

with $\rho$ the density, $F_{\text {conv }}$ the convective energy flux, $C_{\mathrm{P}}$ the heat capacity at constant pressure, $T$ the temperature, $l_{\mathrm{m}}$ the mixing length and $g$ the gravitational acceleration. In the following, we will estimate all quantities in Eq. (8) with the representative quantities inside the star. We have

$F_{\text {conv }} \sim \frac{L_{2}}{4 \pi R_{2}^{2}}, \quad \rho \sim \frac{3 M_{2}}{4 \pi R_{2}^{3}}, \quad T \sim \frac{\mu}{\mathcal{R}} \frac{G M_{2}}{R_{2}}$,

$C_{\mathrm{P}} \sim \frac{5}{2} \frac{\mathcal{R}}{\mu}, \quad \sqrt{g H_{\mathrm{p}}}=\sqrt{\frac{\mathcal{R}}{\mu} T} \sim \sqrt{\frac{G M_{2}}{R_{2}}}$,

$g \sim \frac{G M_{2}}{R_{2}^{2}}$

with $L_{2}$ the luminosity of the donor star, $\mathcal{R}$ the gas constant and $\mu$ the mean molecular weight. Inserting these expressions in Eq. (8) and solving for $\left(\nabla-\nabla_{\text {ad }}\right)$, we obtain

$\nabla-\nabla_{\mathrm{ad}}=\left(\frac{2 \sqrt{2}}{15}\right)^{2 / 3} \frac{L_{2}^{2 / 3} R_{2}^{5 / 3}}{G M_{2}^{5 / 3}}\left(\frac{l_{\mathrm{m}}}{H_{\mathrm{P}}}\right)^{-4 / 3}$

Combining Eqs. (10) and (11), it is straightforward to show that $H_{\mathrm{P}} \sim R_{2}$. While this may be a rather crude approximation, it results from the assumption of considering only average properties within the cool companion. The sound speed in the interior can be evaluated as

$v_{\mathrm{s}}=\sqrt{\frac{G M_{2}}{R_{2}}} \cdot t$

The mixing length is assumed to be of the order of the pressure scale height $H_{\mathrm{P}}$. We here parametrize it as $H_{\mathrm{P}}=\epsilon_{\mathrm{H}} R_{2}$, though we will for simplicity assume $\epsilon_{\mathrm{H}}=1$. The Rossby number can thus be evaluated as

$\mathrm{Ro}=11.5 \frac{v_{\mathrm{c}} P_{\mathrm{Kep}}}{\epsilon_{\mathrm{H}} R_{2}} \frac{R_{\odot}}{\mathrm{km} \mathrm{s}^{-1} \mathrm{yr}}$. 
Inserting this into Eq. (1), we obtain

$$
P_{\text {cycle }}=\left(11.5 \frac{v_{\mathrm{c}} P_{\mathrm{Kep}}}{\epsilon_{\mathrm{H}} R_{2}} \frac{R_{\odot}}{\mathrm{km} \mathrm{s}^{-1} \mathrm{yr}}\right)^{-2 \alpha} P_{\text {rot }} \text {. }
$$

Inserting Eqs. (12) and (13) into Eq. (15), we obtain

$$
\begin{aligned}
P_{\text {cycle }}=P_{\text {rot }} & \left(11.5\left(\frac{2 \sqrt{2}}{15}\right)^{1 / 3} \frac{R_{\odot}}{\mathrm{yr}}\right)^{-2 \alpha} \\
& \times\left(\frac{L_{2}^{2 / 3} R_{2}^{2 / 3}}{M_{2}^{2 / 3}}\left(\frac{l_{\mathrm{m}}}{H_{\mathrm{P}}}\right)^{-4 / 3}\left(\frac{P_{\mathrm{Kep}}}{\epsilon_{\mathrm{H}} R_{2}}\right)^{2}\right)^{-\alpha} .
\end{aligned}
$$

In the following section, we will describe the potential implications for the mass transfer as a result of Applegate's model, and subsequently pursue a systematic comparison of our model with the available data.

\section{Mass transfer as a result of Applegate's scenario}

As well-known from Applegate's model, the presence of a dynamo in the stellar interior can have a relevant impact on the internal structure, affecting the angular momentum distribution and the stellar quadrupole moment (Applegate \& Patterson 1987). As we have shown already in the previous section, the systems considered here are very likely to exhibit such a dynamo mechanism, both due to their rapid rotation as a result of tidal locking, as well as the high convective velocities in the giant star phase. As the donor stars also fill the Roche lobe, we expect that the mass transfer rate in the system will be particularly susceptible even to small changes in the stellar structure, thus implying cyclic changes on the timescale of the stellar dynamo.

In the following, we adopt the finite shell framework for Applegate's model, considering the angular momentum exchange between a finite outer shell and the inner part of the star, as originally derived by Brinkworth et al. (2006) and subsequently extended by Völschow et al. (2016). In this framework, the relative period change $\Delta P / P_{\text {orb }}$, with $P_{\text {orb }}$ the period of the binary, is given as

$$
\begin{aligned}
\frac{\Delta P}{P_{\text {orb }}}= & 0.91 \times 10^{-7}\left(\frac{\Delta E}{E_{\text {sec }}}\right)\left(\frac{a}{R_{\odot}}\right)^{-2}\left(\frac{M_{2}}{M_{\odot}}\right)^{-2} \\
& \times\left(\frac{R_{2}}{R_{\odot}}\right)^{3}\left(\frac{P_{\text {cycle }}}{\mathrm{yr}}\right)\left(\frac{L_{2}}{L_{\odot}}\right) .
\end{aligned}
$$

Here $\Delta E$ denotes the required energy for the structural changes inside the star within one dynamo period $P_{\text {cycle }}, E_{\text {sec }}$ is the energy produced inside the star during that time, $R_{2}=R_{\text {Roche }}$ the radius of the secondary and $L_{2}$ its luminosity, which can be estimated from the effective temperature $T_{\text {eff }}$ and the Stefan-Boltzmann constant $\sigma_{\mathrm{SB}}$ as

$$
L_{2}=4 \pi \sigma_{\mathrm{SB}} R_{\text {Roche }}^{2} T_{\text {eff }}^{4} \text {. }
$$

From Eq. (17), we see that the strength of the Applegate effect is limited by the amount of energy that is available for structural changes within the star, with a maximum ratio of $\Delta E / E_{\mathrm{sec}}=1$. The latter will be adopted in the following to derive an upper limit on the potential effects. These results can be linearly rescaled to account for other possible ratios.

To assess the implications for the stellar interior, we adopt the formula (Applegate 1992; Völschow et al. 2016)

$$
\frac{\Delta P}{P_{\text {orb }}}=-\frac{9 \Delta Q}{a^{2} M_{2}}
$$

with $\Delta Q$ the change in the quadrupole moment that is driving the period variation. Assuming that the initial quadrupole moment of the donor is approximately $Q \sim M_{2} R_{a} R_{b}$, with $R_{a}$ and $R_{b}$ the extension of the donor along two axis and at least approximately $R_{a} \sim R_{b} \sim R_{\text {Roche }}$, a change by $\Delta R$ along one axis will lead to a change

$\Delta Q=M_{2} R_{\text {Roche }} \Delta R$,

implying that

$\Delta R=\frac{\Delta Q}{M_{2} R_{\text {Roche }}}$.

To estimate the mass that is transported to the primary as a result of the change $\Delta R$, we calculate the effective cross section of the flow as (Ritter 1988)

$A_{\mathrm{eff}}=\frac{2 \pi R T_{\mathrm{eff}} R_{2}^{3}}{G M_{2} \mu} F\left(q^{-1}\right)$,

with $\mathcal{R}$ the gas constant and $\mu$ the mean molecular weight, for which we adopt a value of two assuming predominantly ionized gas. As stated by Ritter (1988), the function $F$ can be evaluated as

$F\left(q^{-1}\right)=1.23+0.5 \log \left(q^{-1}\right)$

for $0.5 \leq q^{-1} \leq 10$. We derive an upper limit on the potential mass transferred to the primary by considering the volume $A_{\text {eff }} \Delta R$, multiplied with the density $\rho_{\text {Roche }}$ at $R_{2} \sim R_{\text {Roche }}$. We assume here a typical envelope structure similar to AGB stars, where a small mass $M_{\text {core }}$ forms the central core, while most of the stellar mass is within the envelope $M_{\text {env }} \sim M_{2}$. A typical power-law density profile is then given as (Soker 1992; Kashi \& Soker 2011; Schleicher \& Dreizler 2014)

$\rho(r)=A r^{-2}$,

with $A=\left(M_{2}-M_{\text {core }}\right) /\left(4 \pi R_{\text {Roche }}\right)$. The mean density in the envelope is then given as

$\bar{\rho}=\frac{M_{2}-M_{\text {core }}}{\frac{4 \pi}{3} R_{\text {Roche }}^{3}}$,

while the density at $R_{\text {Roche }}$ follows from

$\rho\left(R_{\text {Roche }}\right)=A R_{\text {Roche }}^{-2}=\frac{M_{2}-M_{\text {core }}}{4 \pi R_{\text {Roche }}^{3}}=\frac{1}{3} \bar{\rho}$.

As a result, the mass transported through the effective area due to the change $\Delta R$ is given as

$\Delta M=A_{\text {eff }} \Delta R \frac{1}{3} \bar{\rho} \sim \frac{1}{3} A_{\text {eff }} \Delta R \frac{M_{2}}{\frac{4 \pi}{3} R_{\text {Roche }}^{3}}$,

where in the last step we assumed that the core mass will be negligible. The average mass transfer rate during one activity period $P_{\text {cycle }}$ is then

$\dot{M}=\frac{\Delta M}{P_{\text {cycle }}}$.

We note that this presents an estimate for the maximum averaged magnitude of the mass transfer rate based on cyclic variations of the stellar quadrupole moment as predicted in Applegate's model, specifically employing the framework outlined by Völschow et al. (2016). This mechanism thus corresponds to a 
D. R. G. Schleicher \& R. E. Mennickent: The dynamo cycle in DPVs

Table 1. Physical parameters of the systems in our sample.

\begin{tabular}{cccccccc}
\hline \hline Binary & $\begin{array}{c}M_{1} \\
{\left[M_{\odot}\right]}\end{array}$ & $\begin{array}{c}M_{2} \\
{\left[M_{\odot}\right]}\end{array}$ & $q$ & $\begin{array}{c}R_{2} \\
{\left[R_{\odot}\right]}\end{array}$ & $\begin{array}{c}T_{2} \\
{[\mathrm{~K}]}\end{array}$ & $\begin{array}{c}P_{\text {orb }} \\
{[\mathrm{d}]}\end{array}$ & $\begin{array}{c}\text { Cycle } \\
{[\mathrm{d}]}\end{array}$ \\
\hline U Cep & 4.938 & 3.22 & 0.652 & 7.05 & 3535 & 3.38 & $515-26663$ \\
UX Mon & 3.38 & 3.9 & 1.15 & $9.95^{*}$ & 5990 & 5.90 & no $^{1}$ \\
DQ Vel & 7.3 & 2.2 & 0.31 & 8.4 & 9350 & 6.08 & 189 \\
V448 Cyg & 24.7 & 13.7 & 0.55 & $16.17^{*}$ & 20340 & 6.52 & no \\
CX Dra & 7.3 & 1.7 & 0.23 & $13.35^{*}$ & 6500 & 6.70 & yes \\
TT Hya & 2.77 & 0.63 & 0.23 & $4.3^{*}$ & 4600 & 6.95 & $?$ \\
iDPV & 9.1 & 1.9 & 0.21 & 8.9 & 12900 & 7.24 & 172 \\
V393 Sco & 7.8 & 2.0 & 0.25 & 9.4 & 7950 & 7.71 & 253 \\
LP Ara & 9.8 & 3.0 & 0.30 & 15.6 & 9500 & 8.53 & 273 \\
V360 Lac & 7.45 & 1.21 & 0.16 & $9.64^{*}$ & 6000 & 10.09 & 322.2 \\
AU Mon & 7.0 & 1.2 & 0.17 & 10.1 & 5750 & 11.11 & 421 \\
BR CMi & 2.31 & 0.14 & 0.06 & $5.54^{*}$ & 4655 & 12.92 & no \\
$\beta$ Lyr & 13.2 & 3.0 & 0.23 & 15.2 & 13200 & 12.94 & 282.4 \\
HD 170582 & 9.0 & 1.9 & 0.21 & 15.6 & 8000 & 16.87 & 537 \\
RX Cas & 5.6 & 1.8 & 0.32 & $23.9^{*}$ & 4400 & 32.31 & 516.1 \\
V495 Cen & 5.85 & 0.97 & 0.17 & 19.7 & 5000 & 33.49 & 1283 \\
SX Cas & 5.1 & 1.5 & 0.29 & $24.42^{*}$ & 4000 & 36.56 & $?$ \\
\hline
\end{tabular}

Notes. The table includes the primary and secondary masses $M_{1}$ and $M_{2}$, the mass ratio $q=M_{2} / M_{1}$, the secondary radius $R_{2}$, the effective temperature of the secondary $T_{2}$, the orbital period $P_{\text {orb }}$ as well as the available information on the presence of a cycle period. We note that radii marked with * have been calculated using the Roche lobe formula (Eq. (4)). Information regarding the presence and duration of a cycle period are based on Harmanec et al. (2015) and Mennickent et al. (2016a). Information for the highly active system U Cep are based on Manzoori (2008). The symbol ${ }^{* *}$ refers to the full name OGLE 05155332-6925581 of iDPV. ${ }^{(1)}$ While Harmanec et al. (2015) still reported the likely presence of a long cycle in UX Mon, the latter is put in doubt based on recent results by Mennickent et al. (2016a), as it cannot be seen in a long baseline. We therefore consider it here as a system without a long cycle.

modulation of the mass transfer rate and can give rise to a cyclic variation due to the accretion luminosity, as

$L_{\mathrm{acc}}=\frac{G M_{1} \dot{M}}{R_{1}}$

with $R_{1}$ the radius of the primary star and $\dot{M}$ the accretion rate. We note that such a relation holds rather independently of whether the accretion luminosity is released via a hot spot or a hot belt, as proposed by Bisikalo \& Kononov (2010), as the released energy is determined by the released energy from the gravitational potential. The variation occuring on the cycle period thus needs to be discriminated from other variations occuring due to binary evolution, typically occuring on timescales of a few $100 \mathrm{yr}$ in the regime of strong interactions. We also note that the values given here correspond to averages, therefore the actual variation at a given time can be smaller or larger.

\section{Comparing model and observations}

In the following, we compare the proposed dynamo model with the available observational data. For this purpose, we present a sample of DPV systems with known physical parameters in Sect. 4.1, which are compared to our model predictions in Sect. 4.2 .

\subsection{Systems with known physical parameters}

To test the model outlined above, we need to construct a sample of DPVs with known physical parameters. The largest such sample has been compiled by Mennickent et al. (2016a), including masses, radii and effective temperatures both for the donor and the gainer star, in the systems LP Ara, iDPV (OGLE
05155332-6925581), HD 170582, V393 Sco, DQ Vel, AU Mon and V360 Lac. We also include $\beta$ Lyr in our analysis, even though previously classified as a W Serpentis system, using the cycle period given by Harmanec et al. (2015). Similarly detailed data were recently obtained for the system V495 Cen (Rosales et al. 2017).

From the Harmanec et al. (2015) sample, we further include all additional systems with measured cycle periods, that is, RX Cas and TT Hya, as well as the systems SX Cas, UX Mon, CX Dra, V448 Cyg and BR CMi, where the presence of a cycle is not clear or the cycle period is not yet measured. For these systems, we note that only the stellar masses and orbital periods were directly measured. The radius of the donor star is therefore calculated from the Roche lobel formula (Eq. (4)). The effective temperature of the donor stars of RX Cas and SX Cas is adopted from Mennickent et al. (2016a, and references therein), the one of TT Hya based on Miller et al. (2007, see also similar results by Etzel 1988; van Hamme \& Wilson 1993), the one of CX Dra based on Guinan et al. (1984, see also earlier work by Koubsky et al. 1980), that of V448 Cyg was based on Djurašević et al. (2009), and that of BR CMi was determined by Harmanec et al. (2015).

As an additional interesting object, we further include the short-period binary U Cep, which was initially investigated and analyzed by Hall (1975) and more recently for instance by Manzoori (2008). As described by Manzoori (2008), it is a very well known and the presumably most active Algol-type binary, for which they inferred 16 characteristic periods with a Fourier analysis of the data, ranging from 1.41 up to $73.05 \mathrm{yr}$. While Hall (1975) considered mass loss as the main driver of the variations, Manzoori (2008) considers both mass loss and magnetic activity as relevant mechanisms, and also Applegate's model is specifically mentioned in the interpretation. While overall this system 
Table 2. Comparison of model predictions with observational results assuming $\epsilon_{\mathrm{H}}=1, l_{\mathrm{m}} / H_{\mathrm{P}}=1$ and $\alpha=0.31$.

\begin{tabular}{ccccccc}
\hline \hline Binary & $\left(P_{\text {cycle }} / P_{\text {orb }}\right)_{\text {obs }}$ & $\left(P_{\text {cycle }} / P_{\text {orb }}\right)_{\text {model }}$ & $P_{\text {cycle,model }} / P_{\text {cycle,obs }}$ & $\left(\Delta P / P_{\text {orb }}\right)_{\max }$ & $\left(\Delta R / R_{2}\right)_{\max }$ & $\dot{M}_{\text {max }}\left[M_{\odot} \mathrm{yr}^{-1}\right]$ \\
\hline U Cep & $152-7889$ & 139 & max. 0.92 & $1.2 \times 10^{-7}$ & $6.7 \times 10^{-8}$ & $8.4 \times 10^{-10}$ \\
UX Mon & - & 57.7 & - & $1.7 \times 10^{-6}$ & $1.4 \times 10^{-6}$ & $5.7 \times 10^{-9}$ \\
DQ Vel & 31.1 & 40.4 & -3 & $3.0 \times 10^{-6}$ & $4.2 \times 10^{-6}$ & $5.1 \times 10^{-8 *}$ \\
V448 Cyg & - & 25.6 & - & $3.0 \times 10^{-5}$ & $3.1 \times 10^{-5}$ & $1.7 \times 10^{-6}$ \\
CX Dra & - & 42.0 & - & $3.3 \times 10^{-5}$ & $2.0 \times 10^{-5}$ & $1.9 \times 10^{-7}$ \\
TT Hya & - & 44.5 & 1.1 & $4.2 \times 10^{-7}$ & $1.3 \times 10^{-6}$ & $2.6 \times 10^{-9}$ \\
iDPV & 23.8 & 27.1 & 1.2 & $1.3 \times 10^{-5}$ & $2.1 \times 10^{-5}$ & $4.4 \times 10^{-7 *}$ \\
V393 Sco & 32.8 & 39.4 & 1.2 & $3.1 \times 10^{-6}$ & $4.8 \times 10^{-6}$ & $4.3 \times 10^{-8 *}$ \\
LP Ara & 32 & 38.6 & 1.0 & $1.7 \times 10^{-5}$ & $1.3 \times 10^{-5}$ & $2.1 \times 10^{-7 *}$ \\
V360 Lac & 31.9 & 32.5 & 1.0 & $4.4 \times 10^{-6}$ & $1.2 \times 10^{-5}$ & $7.1 \times 10^{-8 *}$ \\
AU Mon & 37.9 & 36.6 & - & $1.6 \times 10^{-6}$ & $7.8 \times 10^{-6}$ & $3.4 \times 10^{-8 *}$ \\
BR CMi & - & 22.0 & 0.91 & $1.1 \times 10^{-4}$ & $8.5 \times 10^{-5}$ & $1.9 \times 10^{-7}$ \\
B Lyr & 21.8 & 20.0 & 0.74 & $3.3 \times 10^{-5}$ & $5.6 \times 10^{-5}$ & $4.9 \times 10^{-6 *}$ \\
HD 170582 & 31.8 & 23.6 & 1.4 & $2.4 \times 10^{-5}$ & $3.3 \times 10^{-5}$ & $2.0 \times 10^{-7 *}$ \\
RX Cas & 16 & 23.0 & 0.45 & $6.6 \times 10^{-4}$ & $3.2 \times 10^{-4}$ & $7.8 \times 10^{-7 *}$ \\
V495 Cen & 38.3 & 17.1 & - & $4.5 \times 10^{-5}$ & $6.3 \times 10^{-5}$ & $2.3 \times 10^{-7}$ \\
SX Cas & - & 21.4 & & & &
\end{tabular}

Notes. The table includes the observed and predicted ratios of cycle to orbital period, the ratio between the predicted and observed cycle, the maximum orbital period variation due to magnetic activity, the relative maximum change in the donor radius due to magnetic activity as well as the maximum modulation of the mass transfer rate due to magnetic activity. We note that, when available, we used the observed cycle period in the calculation of the mass transfer rate, while otherwise employing the model prediction. These cases are marked below with an asterisk.

is certainly more active than the typical systems studied here, we still include it in our sample to investigate whether some of its periods could be due to magnetic activity. The pysical parameters of these systems are summarized in Table 1.

\subsection{Comparing observed cycles and predictions}

In the following, we apply our dynamo model to the observed systems in the sample presented in Table 1 . As already mentioned above, we adopt here $\epsilon_{\mathrm{H}}=1$ and $l_{\mathrm{m}} / H_{\mathrm{P}}=1$ for simplicity. The remaining parameter $\alpha$ is then chosen as $\alpha=0.31$, yielding a rather good agreement of the observational data with the average population. Our value of $\alpha$ lies within the range of possible values given by Soon et al. (1993) and Baliunas et al. (1996), and, similar to the observed scaling relations by Saar \& Brandenburg (1999), it corresponds to the somewhat lower range of the parameter space.

However we note here that this parameter combination is not unique, and that one could determine appropriate values of $\alpha$ also for other values of $\epsilon_{\mathrm{H}}$ and $l_{\mathrm{m}} / H_{\mathrm{P}}$, while there are certainly parameter combinations that would lead to larger deviations from the observed sample. While we adopt the above choice for definiteness, our main purpose is to show that there is a parameter space for which the model and the data are about consistent.

The results for the model are given in Table 2, and a comparison of the observed and predicted ratios between the long cycle (as determined from Eq. (16)) and the orbital period are shown as a function of the orbital period in Fig. 1. We note that from our sample of 17 binary stars with well-determined physical parameters, only ten have a well-measured long cycle. The observational data for the period ratio thus appear to be roughly consistent with a flat relation, with a mean value of 29.7. Model predictions were made for all 17 binaries. If we do not consider

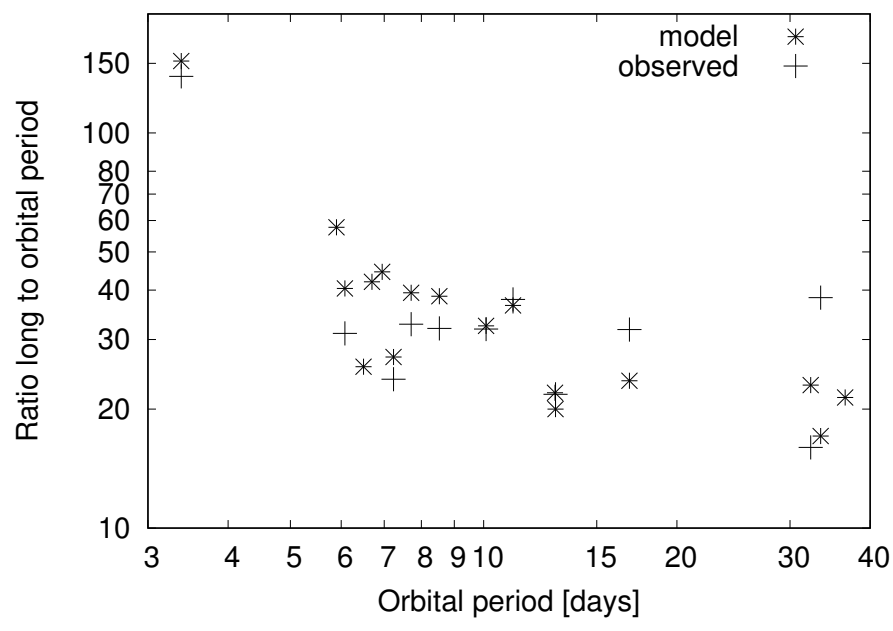

Fig. 1. Ratio of the long to the orbital period as a function of the orbital period based on the observed data as well as our model. The corresponding data are given in Tables 1 and 2. For the system U Cep, we adopted the lowest observed period in the comparison.

the highly extraordinary system U Cep, the mean value obtained from the models is about 32, with U Cep it is about 38 .

Focusing first on the binaries where both model predictions and observational data are available, we find a relatively good agreement. In particular, for the seven binaries with orbital periods between five and 13 days, the maximum deviation between predicted and observed ratio is $30 \%$, with the average deviation of order $12 \%$. For the three binaries with longer orbital periods, the deviation ranges from $26 \%$ to about $55 \%$. Overall, the ratio of long to orbital period appears to slightly decrease with orbital period in the models, while there is no such clear trend in the 
D. R. G. Schleicher \& R. E. Mennickent: The dynamo cycle in DPVs

observational data. We however do note that a clear conclusion cannot be drawn due to the low number statistics.

As mentioned above, the system $\mathrm{U}$ Cep is a special case, due to its strong activity and the many different periods that were inferred by Manzoori (2008). Our model with the parameters given above would predict an activity cycle of about $1.29 \mathrm{yr}$, which is comparable to the lowest period inferred by Manzoori (2008) of $1.41 \mathrm{yr}$. If that is the right period to compare with, the difference between observed and predicted cycle is about $8 \%$. However, the comparison clearly needs to be treated with caution, due to the many cycles found in the system and the generally strong activity, which will certainly involve other mechanisms as well.

For the verification of our model, we further compare its predictions to the numerical simulation by Blackman et al. (2001), to our knowledge the only numerical simulation so far where the non-linear dynamo phase has been modeled for an AGB star. While their model includes strong differential rotation, we expect here a different regime of rather rigidly rotating stars as a result of strong tidal interaction. Nevertheless, a comparison may be instructive to see if the order of magnitude is about right. They consider an AGB star with $3 M_{\odot}$, a radius of $4.3 R_{\odot}$, a luminosity of about $20 L_{\odot}$ and a rotation period of about 14 days around the convection zone. Inserting these numbers in our model, we obtain an expected ratio of about 33.3, while the ratio found in their simulations corresponds to a value of 10.4. From this comparison, we may thus conclude that the accuracy of our model lies within about a factor of three. Still, we note that such a comparison has to be treated with caution, due to uncertainties present even in the modeling of the solar dynamo. In the limits of such uncertainties, we may cautiously conclude that a dynamo mechanism can potentially produce long periods similar to the observed ones, though of course further investigation is necessary.

In Table 2, we further provide predictions based on Applegate's model for the maximum ratios $\Delta P / P_{\text {orb }}$ due to magnetic activity, with typical values of $10^{-5}-10^{-6}$. The highest possible value is found in the system V495 Cen, with a maximum ratio of $6.6 \times 10^{-4}$. We note that due to the low magnitude of these variations and as these are upper limits, a direct comparison with observations is however difficult, as we cannot separate the phenomenon from other effects like binary evolution, which also produces period variations and a time-dependence of the mass transfer rate. Similarly, the maximum variation in the relative stellar radius due to magnetic activity is found to be of the order of $10^{-5}-10^{-6}$, again with a maximum value of $3.2 \times 10^{-4}$ in case of V495 Cen. The predicted modulations of the mass transfer rate range from a maximum of $1.9 \times 10^{-6} M_{\odot} \mathrm{yr}^{-1}(\beta$ Lyr $)$ to a minimum value of $2.6 \times 10^{-9} M_{\odot} \mathrm{yr}^{-1}$ (TT Hya).

For the highly active system $\mathrm{U}$ Cep, it is interesting to note that our model predicts a rather weak influence of the Applegate mechanism, with period variations $\Delta P / P_{\text {orb }}$ of the order $10^{-7}$ and a modulated accretion rate of the order $10^{-9} M_{\odot} \mathrm{yr}^{-1}$. The latter provides another indication that Applegate's model is probably not the main reason for the activity in that system. As mentioned above, these values correspond to an average over the dynamo cycle, and the actual variations at a given time may be higher than the average. We note, though, that the value of the average corresponds to an upper limit.

As discussed in the previous section, the presence of a long cycle is not always fully clear. As reported by Harmanec et al. (2015), the systems V448 Cyg and BR CMi show no signs of such a cycle at this point. We note that these systems appear as somewhat unusual. In particular, the donor in V448 Cyg has a very high mass of $13.7 M_{\odot}$, while the donor in BR CMi has a very low mass of $0.14 M_{\odot}$. These systems thus correspond to the extreme cases for the donor mass range considered here, and it is at least conceivable that in this physical regime, the dynamo process is different or other stellar processes are more important. In case of BR CMi, an additional concern is the very low mass ratio of about 0.06, implying a large Lubow-Shu critical radius. While most DPVs were previously found to be tangentialimpact systems (Mennickent et al. 2016a), this system would be more likely to form a disk, implying a different energy dissipation mechanism. The latter is potentially more gradual, and may thus wash out some of the originally present fluctuations due to the dynamo. The recent investigation by Mennickent et al. (2016a) found that a constant orbital period reproduces well the light curve of UX Mon over $57 \mathrm{yr}$, casting doubts about the previously reported period changes for this star. Moreover, they found no evidence for a long cycle. We note that all three of these systems have rather extreme mass ratios, which can potentially be relevant for the interpretation.

For the systems TT Hya and SX Cas, the presence of a long cycle is unclear, but not strongly ruled out. Here, our model predicts typical ratios of the long to orbital period of 41.5 and 17.1, respectively. The expected modulation of the accretion rate in TT Hya is quite on the low side, with $2.6 \times 10^{-9} M_{\odot} \mathrm{yr}^{-1}$, while one expects about $2.3 \times 10^{-7} M_{\odot} \mathrm{yr}^{-1}$ for SX Cas. These relatively low values may partly contribute to the difficulty to detect a long cycle, though we encourage additional observations in particular for SX Cas to either confirm a long cycle or to provide stronger constraints. Finally, we note that for the system CX Dra, indications for a long cycle are present, but its duration is not well constrained. A better determination of the cycles will thus be valueable for comparison with the model predictions given here.

\section{Discussion and conclusions}

In this manuscript, we have considered the magnetic activity as a potential explanation for the long period in DPVs, which exhibit cyclic light variations on a timescale an order of magnitude larger than the corresponding orbital time scale, many of them with a characteristic ratio of about $3.5 \times 10^{1}$ (Mennickent et al. 2003). As a result of efficient synchronization via dynamical tides, we expect rapid rotation of the donor star, with its rotation period equal to the period of the binary system (Tassoul 1987; Zahn 1989; Zahn \& Bouchet 1989). The resulting rapid rotation, corresponding to $10-30 \%$ of the Keplerian value, provides ideal conditions to drive a magnetic dynamo.

To estimate the dynamo cycle within the star, we have adopted the relation proposed by Soon et al. (1993) and Baliunas et al. (1996) between the activity cycle and the orbital period, and estimated the dynamo number in the stellar interior. Based on Applegate's mechanism, particularly the formulation provided by Völschow et al. (2016), we further estimated the impact of the magnetic dynamo on the stellar interior, including changes in the quadrupole moment, the stellar radius and the resulting modulation of the mass transfer rate of the star. This mechanism is known to produce orbital period variations in magnetically active binaries, and we suggest here that it can also lead to a time-variable mass transfer rate in semi-detached systems, such as the DPVs. We have shown that the expected orbital period variations are negligible for the DPVs, consistent with the observational results, thus leaving the variable mass transfer as the main relevant observable.

This model has been applied to a sample of close binaries with at least one massive star and known physical parameters, 
including the DPV candidates outlined by Mennickent et al. (2016a) and additional systems described by Harmanec et al. (2015). In particular for the systems with rotation periods between 5 and 13 days, we find a good agreement with our model, with average deviations of the long cycle of the order $12 \%$, while larger variations up to $55 \%$ are possible for longer rotation periods. This match is obtained using the same basic assumptions for the whole set of targets and with only one adjustable parameter, $\alpha$. As an additional estimate of our model uncertainties, we have compared it to the numerical simulation of an AGB dynamo by Blackman et al. (2001), to date unfortunately the only numerical simulation investigating the non-linear phase of the dynamo in this regime, finding an uncertainty of about a factor of 3. Despite the uncertainties both in the model as well as within the dynamo simulations, it shows that the required orders of magnitudes can be produced via a dynamo mechanism.

Particularly interesting are the objects where no long cycle has been found to date, including V448 Cyg and BR CMi (Harmanec et al. 2015). These objects are at the extreme ends for the masses of the donor stars, with $13.7 M_{\odot}$ for V448 Cyg and $0.14 M_{\odot}$ for BR CMi. In case of BR CMi, we further note that the low mass ratio of 0.06 implies a large Lubow-Shu critical radius, potentially leading to the formation of an extended disk around the primary star. In case of TT Hya and SX Cas, the presence of a long cycle is also unclear, but still feasible. In case of UX Mon, the analysis by Mennickent et al. (2016a) is also not compatible with a long cycle. We note that all three of these systems have rather extreme mass ratios, which may be relevant for the interpretation. The system CX Dra shows signs of a long cycle, but the duration is not well constrained, therefore limiting the possibility of a model comparison. We also note that in a few cases, the long cycle has turned out to be non-periodic. The most extreme case is LMC SC6 57364. Mennickent et al. (2005) found that between JD 2448800 and 2450000 , the long term period was 340 days, while it shortened to 270 days around JD 2450500 , implying a variability amplitude of $20 \%$. Poleski et al. (2010) report for this object $\mathrm{d} P_{2} / \mathrm{d} t=-0.01724 \pm$ 0.00040 . Such variability is also known from the magnetically active RS CVn stars (Lindborg et al. 2013), and activity variations are known also from solar analogues (Käpylä et al. 2016), and are thus generally consistent with the idea of the long cycle being driven by a dynamo mechanism.

In general, we should note that the current number of objects in our sample with known physical parameters and known long cycles is only 11 , thus limiting the ability to identify clear trends in the observational sample and limiting the comparison with model predictions. In addition, even within these systems, the physical parameters are not always well constrained, and further work on their characterization is certainly needed. Even in well-studied systems like AU Mon with a very good eclipsing light curve from CoRoT and very systematic ground-based $V$ photometry (Lorenzi 1980b; Desmet et al. 2010), minor uncertainties are present in the physical parameters. For instance, Desmet et al. (2010) have solved the light curve assuming a semi-detached configuration and interpreting the residuals from the fit as due to short-periodic oscillations, finding a radius of $5.6 \pm 0.5 R_{\odot}$ for the more massive star. Djurašević et al. (2010) solved the same light curve assuming also a flat accretion disk including three spots, yielding a radius of $5.1 \pm 0.5 R_{\odot}$, thus roughly consistent within the errors. Mimica \& Pavlovski (2012) instead assumed a clumpy and asymmetric accretion disk, and Atwood-Stone et al. (2012) considered additional contributions from the gas stream. All of these studies are however based on the mass ratio of $0.17 \pm 0.03$ estimated by Desmet et al. (2010), which limits the accuracy that can be reached at this point. In other few systems, the mass of the B-type star is inferred from the spectral type assuming a main-sequence classification (Mennickent et al. 2016a).

We also investigated the short period binary U Cep (Hall 1975; Manzoori 2008), as it has well-determined physical parameters and is the perhaps most active Algol-type system known to date. We found that our model can potentially explain the shortest period inferred in that system, even though the predicted magnitude of the period variation as well as the modulation of the accretion rate are rather weak. It is thus conceivable that the activity in the system is mostly driven by mass loss, as originally proposed by Hall (1975).

In this paper we have shown that the DPV long cycles could be caused by changes in the donor radius driven by the Applegate mechanism; consistently they should produce cyclic mass transfer variations potentially observable by changes in the accretion luminosity. We notice however that due to the presence of the disk, the gas stream cannot hit directly the star, but hits the disk at its outer edge, producing a hotspot or hotline, which is revealed in light curve models (Mennickent \& Djurašević 2013) and hydrodynamical simulations (Bisikalo et al. 1998, 1999, 2003). It is then possible that an increase of the energy released at the hotspot produces a stronger hotspot wind at long cycle maximum, as modeled by van Rensbergen et al. (2008). This strengthened wind can be seen as the extra light emitting source reported for instance in V393 Scorpii (Mennickent et al. 2012b), DQ Velorum (Barría et al. 2014) and $\beta$ Lyrae (Harmanec et al. 1996; Hoffman et al. 1998; Ak et al. 2007). In this case the hotspot should be brighter at long cycle maximum, something revealed by Doppler tomography in the DPV HD 170582 (Mennickent et al. 2016b). More work is needed in this line to firmly validate our proposed scenario.

The possibility of driving dynamos in Algol-type systems is nothing new, and has already been proposed by Sarna et al. (1997) and Soker (2002). Resulting variations of the mass transfer rate have been suggested by Bolton (1989) and Meintjes (2004), considering various mechanisms, including the direct impact of the magnetic field on the stellar surface and the stream as well as the potential presence of cool spots due to rising magnetic bubbles, which may interact with the mass stream and change both the densities and temperatures close to L1. Here we consider the potential contribution resulting from the Applegate mechanism (Applegate \& Patterson 1987; Völschow et al. 2016), implying cyclic variations of the quadrupole moment, the stellar radius and the mass transfer rate, finding typical values of the modulation of $10^{-7}-10^{-8} M_{\odot} \mathrm{yr}^{-1}$.

Our results have been derived based on simplifying assumptions, considering mean properties of the stellar interior and the approximation of mixing length theory. Also the use of the Roche lobe formula by Paczyński (1971) is a simplifying assumption, as the donor star may flatten as a result of rapid rotation, potentially altering the equipotential surfaces. Within the limit of these uncertainties, it appears at least plausible that a dynamo cycle with properties similar to the observed cycle will emerge, and independent of the details, we certainly expect magnetic activity in these type of systems. A piece of evidence pointing in this direction is the detection of chromospheric activity in the donor of the DPV V393 Scorpii (Mennickent et al. 2012b). For a better understanding of dynamos in these type of systems, it is however necessary to first better understand dynamos in giant and AGB stars. While there is some theoretical work pursued by Sarna et al. (1997) and Soker (2002), the only simulation exploring the non-linear dynamo in AGB stars is based on 
D. R. G. Schleicher \& R. E. Mennickent: The dynamo cycle in DPVs

Blackman et al. (2001), while Dorch (2004) investigated the linear regime including the growth of the magnetic field. It will, however, be desirable to explore further the dependence on various physical parameters, including the amount of rotation and different profiles of the rotational velocity, as well as the masses and structure of the stars. On the long term, one should pursue a joint modeling of the dynamo in the donor star along with the whole binary system (the primary potentially approximated via a point particle), to obtain a better understanding of the dynamical evolution in such situations.

The model proposed here can be tested probing the magnetic activity of the donor star using polarimetry. In addition, one may search for indirect confirmations or constraints. As an example, Mennickent et al. (2016a) discussed 3 RS CVn systems that were previously misidentified as DPVs. While these systems are detached and not in the mode of accretion, a long cycle with very similar properties as in the case of DPVs has been inferred from possible variable surface spots, providing an indirect confirmation of the proposed scenario. An identification of more such systems would thus be desirable in order to probe whether it is indeed a universal phenomenon. Time-dependent measurements of the accretion rates will help to confirm a potential modulation of the accretion rate via dynamo cycles and link the variation more closely to the properties of the donor star. In addition, the extension of such samples with known, well-constrained physical properties of the binaries and known, well-understood long cycles will be crucial to build up better statistics and to more strongly constrain the physical picture driving the long period.

Acknowledgements. We thank the two referees for insightful comments that helped to improve our manuscript. D.R.G.S. thanks for Funding through Fondecyt regular (project code 1161247) and through the "Concurso Proyectos Internacionales de Investigación, Convocatoria 2015" (project code PII20150171). R.E.M. acknowledges support by VRID-Enlace 214.016.002 1.0 and the BASAL Centro de Astrofísica y Tecnologías Afines (CATA) PFB $06 / 2007$.

\section{References}

Ak, H., Chadima, P., Harmanec, P., et al. 2007, A\&A, 463, 233

Applegate, J. H. 1992, ApJ, 385, 621

Applegate, J. H., \& Patterson, J. 1987, ApJ, 322, L99

Atwood-Stone, C., Miller, B. P., Richards, M. T., Budaj, J., \& Peters, G. J. 2012 ApJ, 760, 134

Baliunas, S. L., Nesme-Ribes, E., Sokoloff, D., \& Soon, W. H. 1996, ApJ, 460, 848

Barría, D., Mennickent, R. E., Graczyk, D., \& Kołaczkowski, Z. 2014, A\&A, 567, A140

Bisikalo, D. V., Boyarchuk, A. A., Chechetkin, V. M., Kuznetsov, O. A., \& Molteni, D. 1998, MNRAS, 300, 39

Bisikalo, D. V., Boyarchuk, A. A., Chechetkin, V. M., Kuznetsov, O. A., \& Molteni, D. 1999, Astron. Rep., 43, 797

Bisikalo, D. V., Boyarchuk, A. A., Kaigorodov, P. V., \& Kuznetsov, O. A. 2003 Astron. Rep., 47, 809

Bisikalo, D. V., \& Kononov, D. A. 2010, Mem. Soc. Astron. Ital., 81, 187

Blackman, E. G., Frank, A., Markiel, J. A., Thomas, J. H., \& Van Horn, H. M. 2001, Nature, 409, 485

Böhm-Vitense, E. 2007, ApJ, 657, 486

Bolton, C. T. 1989, Space Sci. Rev., 50, 311

Brinkworth, C. S., Marsh, T. R., Dhillon, V. S., \& Knigge, C. 2006, MNRAS 365,287

Crawford, J. A. 1955, ApJ, 121, 71

de Mink, S. E., Sana, H., Langer, N., Izzard, R. G., \& Schneider, F. R. N. 2014 ApJ, 782, 7

Desmet, M., Frémat, Y., Baudin, F., et al. 2010, MNRAS, 401, 418

Djurašević, G., Vince, I., Khruzina, T. S., \& Rovithis-Livaniou, E. 2009, MNRAS, 396, 1553

Djurašević, G., Latković, O., Vince, I., \& Cséki, A. 2010, MNRAS, 409, 329

Dorch, S. B. F. 2004, A\&A, 423, 1101

Dubé, C., \& Charbonneau, P. 2013, ApJ, 775, 69

Eggleton, P. P., \& Kisseleva-Eggleton, L. 2006, Ap\&SS, 304, 75
Etzel, P. B. 1988, AJ, 95, 1204

Gaposchkin, S. 1944, ApJ, 100, 230

Guinan, E. F. 1989, Space Sci. Rev., 50, 35

Guinan, E. F., Koch, R. H., \& Plavec, M. J. 1984, ApJ, 282, 667

Hall, D. S. 1975, Acta Astron., 25, 1

Harmanec, P., Morand, F., Bonneau, D., et al. 1996, A\&A, 312, 879

Harmanec, P., Koubský, P., Nemravová, J. A., et al. 2015, A\&A, 573, A107

Hoffman, J. L., Nordsieck, K. H., \& Fox, G. K. 1998, AJ, 115, 1576

Kamiński, K. Z., Ruciński, S. M., Matthews, J. M., et al. 2007, AJ, 134, 1206

Käpylä, M. J., Käpylä, P. J., Olspert, N., et al. 2016, A\&A, 589, A56

Kashi, A., \& Soker, N. 2011, MNRAS, 417, 1466

Kippenhahn, R., \& Weigert, A. 1967, ZAp, 65, 251

Kippenhahn, R., Weigert, A., \& Weiss, A. 2012, Stellar Structure and Evolution (Berlin Heidelberg: Springer-Verlag)

Koubsky, P., Harmanec, P., Horn, J., et al. 1980, Bulletin of the Astronomical Institutes of Czechoslovakia, 31, 75

Křiž, S. 1970, Bulletin of the Astronomical Institutes of Czechoslovakia, 21, 211

Kříž, S. 1971, Bulletin of the Astronomical Institutes of Czechoslovakia, 22, 108

Kříž, S. 1972, Bulletin of the Astronomical Institutes of Czechoslovakia, 23, 328

Lanza, A. F., \& Rodonò, M. 1999, A\&A, 349, 887

Lanza, A. F., Rodono, M., \& Rosner, R. 1998, MNRAS, 296, 893

Leal-Ferreira, M. L., Vlemmings, W. H. T., Kemball, A., \& Amiri, N. 2013, A\&A, 554, A134

Lindborg, M., Mantere, M. J., Olspert, N., et al. 2013, A\&A, 559, A97

Lorenzi, L. 1980a, A\&A, 85, 342

Lorenzi, L. 1980b, A\&A, 85, 342

Lorenzi, L. 1980c, A\&AS, 40, 271

Lubow, S. H., \& Shu, F. H. 1975, ApJ, 198, 383

Lucy, L. B. 2005, A\&A, 439, 663

Manzoori, D. 2008, Ap\&SS, 318, 57

Matranga, M., Drake, J. J., Kashyap, V., \& Steeghs, D. 2012, ApJ, 747, 132

Meintjes, P. J. 2004, MNRAS, 352, 416

Mennickent, R. E., \& Djurašević, G. 2013, MNRAS, 432, 799

Mennickent, R. E., Pietrzyński, G., Diaz, M., \& Gieren, W. 2003, A\&A, 399, L47

Mennickent, R. E., Cidale, L., Díaz, M., et al. 2005, MNRAS, 357, 1219

Mennickent, R. E., Assmann, P., \& Sabogal, B. 2006, in Rev. Mex. Astron. Astrofis. Conf. Ser., 26, 173

Mennickent, R. E., Kołaczkowski, Z., Michalska, G., et al. 2008, MNRAS, 389, 1605

Mennickent, R. E., Djurašević, G., Kołaczkowski, Z., \& Michalska, G. 2012a, MNRAS, 421, 862

Mennickent, R. E., Kołaczkowski, Z., Djurasevic, G., et al. 2012b, MNRAS, 427, 607

Mennickent, R. E., Djurašević, G., Cabezas, M., et al. 2015, MNRAS, 448, 1137

Mennickent, R. E., Otero, S., \& Kołaczkowski, Z. 2016a, MNRAS, 455, 1728

Mennickent, R. E., Zharikov, S., Cabezas, M., \& Djurašević, G. 2016b, MNRAS, 461, 1674

Michalska, G., Mennickent, R. E., Kołaczkowski, Z., \& Djurašević, G. 2010, in Binaries - Key to Comprehension of the Universe, eds. A. Prša, \& M. Zejda, ASP Conf. Ser., 435, 357

Miller, B., Budaj, J., Richards, M., Koubský, P., \& Peters, G. J. 2007, ApJ, 656, 1075

Mimica, P., \& Pavlovski, K. 2012, in From Interacting Binaries to Exoplanets: Essential Modeling Tools, eds. M. T. Richards, \& I. Hubeny, IAU Symp., 282, 63

Paczyński, B. 1971, ARA\&A, 9, 183

Pandey, J. C., \& Singh, K. P. 2008, MNRAS, 387, 1627

Pawlak, M., Graczyk, D., Soszyński, I., et al. 2013, Acta Astron., 63, 323

Peters, G. J. 1994, in Interacting Binary Stars, ed. A. W. Shafter, ASP Conf. Ser., 56,384

Peterson, W. M., Mutel, R. L., Güdel, M., \& Goss, W. M. 2010, Nature, 463, 207

Plavec, M. J. 1980, in Close Binary Stars: Observations and Interpretation, eds. M. J. Plavec, D. M. Popper, \& R. K. Ulrich, IAU Symp., 88, 251

Plavec, M. J. 1989, Space Sci. Rev., 50, 95

Plavec, M. J., Weiland, J. L., \& Koch, R. H. 1982, ApJ, 256, 206

Poleski, R., Soszyński, I., Udalski, A., et al. 2010, Acta Astron., 60, 179

Ribeiro, T., Kafka, S., Baptista, R., \& Tappert, C. 2010, AJ, 139, 1106

Ritter, H. 1988, A\&A, 202, 93

Rosales, J. A., Mennickent, R. E., Djurašević, G., et al. 2017, MNRAS, submitted

Rüdiger, G., Elstner, D., Lanza, A. F., \& Granzer, T. 2002, A\&A, 392, 605

Saar, S. H., \& Brandenburg, A. 1999, ApJ, 524, 295

Sarna, M. J. 1993, MNRAS, 262, 534

Sarna, M. J., Muslimov, A., \& Yerli, S. K. 1997, MNRAS, 286, 209 
Schleicher, D. R. G., \& Dreizler, S. 2014, A\&A, 563, A61

Schwope, A. D., Hambaryan, V., Schwarz, R., Kanbach, G., \& Gänsicke, B. T. 2002, A\&A, 392, 541

Soderhjelm, S. 1980, A\&A, 89, 100

Soker, N. 1992, ApJ, 389, 628

Soker, N. 2000, ApJ, 540, 436

Soker, N. 2002, MNRAS, 337, 1038

Soon, W. H., Baliunas, S. L., \& Zhang, Q. 1993, ApJ, 414, L33

Soszyński, I., Pawlak, M., Pietrukowicz, P., et al. 2016, Acta Astron., 66, 405

Tassoul, J.-L. 1987, ApJ, 322, 856

Tutukov, A. V., \& Pavlyuchenkov, Y. N. 2004, Astron. Rep., 48, 800

van Hamme, W., \& Wilson, R. E. 1993, MNRAS, 262, 220

van Rensbergen, W., De Greve, J. P., De Loore, C., \& Mennekens, N. 2008, A\&A, 487, 1129 van Rensbergen, W., de Greve, J. P., Mennekens, N., Jansen, K., \& de Loore, C. 2011, A\&A, 528, A16

Vlemmings, W. H. T., Diamond, P. J., \& van Langevelde, H. J. 2002, A\&A, 394, 589

Vlemmings, W. H. T., van Langevelde, H. J., \& Diamond, P. J. 2005, A\&A, 434, 1029

Vlemmings, W. H. T., Diamond, P. J., \& Imai, H. 2006, Nature, 440, 58

Völschow, M., Schleicher, D. R. G., Perdelwitz, V., \& Banerjee, R. 2016, A\&A, 587, A34

Young, A., \& Snyder, J. A. 1982, ApJ, 262, 269

Zahn, J.-P. 1989, A\&A, 220, 112

Zahn, J.-P., \& Bouchet, L. 1989, A\&A, 223, 112

Zorotovic, M., \& Schreiber, M. R. 2013, A\&A, 549, A95 